\title{
Inherently Improper Surface Parametric Supports
}

\author{
Eng-Wee Chionh ${ }^{1)}$ \\ School of Computing, National University of Singapore, Singapore 117543 \\ Xiao-Shan Gao, Li-Yong Shen \\ KLMM, Institute of Systems Science, AMSS, Chinese Academy of Sciences, \\ China 100080
}

\begin{abstract}
We identify a class of monomial supports from which any rational parametrizations for surfaces are improper. These inherently improper surface parametric supports are called improper supports and they can be characterized by a simple necessary and sufficient condition. The constructive proof of this condition can be implemented to detect and correct improper parametrizations due to improper supports. Our results also extend to rational parametric representations of hypersurfaces with arbitrary dimensions.
\end{abstract}

\section{Introduction}

Rational parametrizations are fundamental curve and surface representations in computer shape modeling and processing. Perhaps the most basic property of a rational parametrization is whether it is proper (one-to-one) or improper (many-to-one). Improper parametrizations are undesirable because the parametric degree could be unnecessarily high. A high degree parametrization costs more to analyze and process. What is worst, operations such as inversion becomes impossible or much more complicated. Thus it is important to be able to detect improper parametrizations efficiently, and when one is detected, to attenuate or get rid of the improperness.

For curves, Lüroth's theorem settles the improper parametrization problem thoroughly. The theorem says that given a curve rational parametrization $(X(t), Y(t))$ with coefficient field $k$, there is a rational function $s(t) \in k(t)$ such that $k(X(t), Y(t))=k(s(t))$. If $s(t)$ is linear in $t$, the given parametrization is already proper. Regardless, there are rational functions $\phi, \psi$ such that $X(t)=\phi(s(t)), Y(t)=\psi(s(t))$. Consequently, the reparametrization $(\phi(s), \psi(s))$ is always proper and generates the same curve. Furthermore, practical algorithms to find $s(t)$ using polynomial gcd, involutions, and elimination are described respectively in $[8,7,4]$.

For surfaces the situation is not so satisfactory. When $k$ is the field of complex numbers a theorem analogous to Lüroth's was proved by Castelnuovo but the proof was nonconstructive. To make matters worse, when confined to rational or real coefficients, a reparametrization similar to that of curves to avoid improperness may not be possible. These facts are surveyed in [6]. Though correcting an improper surface rational parametrization is difficult or impossible, there are practical algorithms to detect one $[2,5]$.

\footnotetext{
1) Email address: chionhew@comp.nus.edu.sg(Eng-Wee Chionh) xgao@mmrc.iss.ac.cn(Xiao-Shan Gao) shenly@amss.ac.cn(Li-Yong Shen)
} 
However, the situation greatly improves, if instead of dealing with the general problem of improper surface parametrizations, we study a class of surface rational parametrizations arising from some special monomial supports. These monomial supports are special because rational parametrizations constructed from them are always improper whatever the coefficients are. To emphasize this characteristic, it seems appropriate to refer to such monomial supports as inherently improper surface parametric supports, or simply improper supports. The main results of the paper are thus two-fold:

- highlighting the existence of inherently improper surface parametric supports, and

- proving a theorem constructively for the detection and correction of improper parametrizations due to improper supports.

As surfaces in the 3-dimensional space are the main objects of interest, the paper focuses on rational parametrizations from $k^{2}$ to $k^{3}$, where in practice the field $k$ is either the field of rational numbers or the real numbers. This also simplifies the notation and derivation without losing generality, for the results can easily be extended to rational parametrizations from $k^{n}$ to $k^{n+1}$ for $n>2$. This is because the algebraic tools of implicitization, the BKK degree bound, Jacobians, and $n$-volume determinantal expressions are all valid in the general $n$-dimensional setting.

The paper developes as follows. Section 2. defines improper indices and support transformations and establishes two basic results: a support transformation is a reparametrization, and a support that can be shrunk is improper. Section 3. proves that the improper index of a support is simply the gcd of the normalized areas of all its triangular (3-point) sub-supports. Section 4. studies how likely an arbitrary surface parametric support is improper. Finally, Section 5. concludes the paper with a summary and suggests possible future research.

\section{Preliminaries}

In this section we introduce all terminology and notation needed for the paper. The most important concepts defined are improper indices and support transformations. Support transformations are important because they are reparametrizations and improper supports can be shrunk by support transformations.

\subsection{Surface Parametric Supports, Newton Polygons, Normalized Areas}

Let $\mathbf{Z}$ and $\mathbf{R}$ be respectively the set of integers and reals. The set $\mathbf{Z} \times \mathbf{Z}$ is the set of lattice points and $\mathbf{R} \times \mathbf{R}$ is the Euclidean plan. A set $S \subseteq \mathbf{Z} \times \mathbf{Z}$ is called a surface parametric support if $S$ is a finite set with at least three lattice points; that is, $3 \leq|S|<\infty$. (Note that the surface becomes degenerate if the lattice points of a surface support are collinear.) For example, the parametric support for the total degree $n \geq 1$ parametrization is

$$
T_{n}=\{(i, j): 0 \leq i, 0 \leq j, i+j \leq n\},
$$

and the parametric support for the bidegree $m \times n$ parametrization is

$$
B_{m, n}=\{(i, j): 0 \leq i \leq m, 0 \leq j \leq n\} .
$$


Let $S$ be a surface parametric support. Any $S^{\prime} \subseteq S$ with $\left|S^{\prime}\right| \geq 3$ will be called a subsupport of $S$. We call a support $S$ triangular if $|S|=3$. Triangular sub-supports turn out to be significant in the study of improper supports.

The Newton polygon $N P(S)$ of a parametric support $S$ is the convex hull of $S$. The normalized area $N A(P)$ of a polygon $P$ is twice the usual Euclidean area of $P$. The normalized area of a surface parametric support $S$ is $N A(N P(S))$. For example,

$$
N A\left(N P\left(T_{n}\right)\right)=n^{2}, \quad N A\left(N P\left(B_{m, n}\right)\right)=2 m n .
$$

\subsection{Shrinking Support Transformations}

Let $T: \mathbf{R} \times \mathbf{R} \rightarrow \mathbf{R} \times \mathbf{R}$ be an invertible affine transformation with $T(x, y)=(a x+$ $b y+c, d x+e y+f)$. The transformation $T$ is a support transformation with respect to a parametric support $S$ if $T(S) \subseteq \mathbf{Z} \times \mathbf{Z}$; that is, if $T(S)$ is also a surface parametric support. The absolute value of the determinant of the Jacobian matrix of $T$ is written

$$
J(T)=a b s\left|\begin{array}{ll}
a & d \\
b & e
\end{array}\right| .
$$

Given a support transformation $T$ for a parametric support $S$, it is a well-known fact in calculus that we have

$$
J(T)=\frac{N A(N P(T(S)))}{N A(N P(S))} .
$$

For obvious reasons, a support transformation $T$ with $J(T)<1$ is called a shrinking support transformation, and a support that admits a shrinking transformation is called a shrinkable support.

\subsection{Improper Indices}

Let $S$ be a surface parametric support. A rational surface can be defined on $S$ as

$$
(X(s, t), Y(s, t), Z(s, t))=\frac{\sum_{(i, j) \in S}\left(x_{i, j}, y_{i, j}, z_{i, j}\right) s^{i} t^{j}}{\sum_{(i, j) \in S} w_{i, j} s^{i} t^{j}}
$$

where $w_{i, j}, x_{i, j}, y_{i, j}, z_{i, j} \in k$ are coefficients from some field $k$. The algebraic degree of the surface (6) with generic coefficients is denoted $A D(S)$. The improper index of $S$ is defined to be

$$
I X(S)=\frac{N A(N P(S))}{A D(S)} .
$$

Since $N A(N P(S))$ is the expected surface degree, or the BKK degree [3], for a surface parametrized on $S$, the improper index $I X(S)$ gives the number of parameter points $(s, t)$ corresponding to a general surface point $(X(s, t), Y(s, t), Z(s, t))$. In other words, $S$ is an improper support if and only if $I X(S)>1$.

2.3..1. Example: $I X(\{(0,0),(2,0),(0,2),(2,2)\})$ 


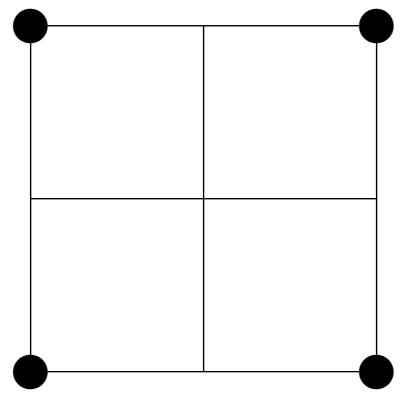

Let parametric support $S=\{(0,0),(2,0),(0,2),(2,2)\}$. By the technique of random coefficients or otherwise, we find $A D(S)=2$. Thus

$$
I X(\{(0,0),(2,0),(0,2),(2,2)\})=\frac{2 \times 2 \times 2}{2}=4 .
$$

Indeed, there are four parameter points $( \pm s, \pm t)$ corresponding to a general surface point $(X, Y, Z)$.

2.3..2. Example: $I X(\{(0,0),(2,0),(1,1),(0,2),(2,2)\})$

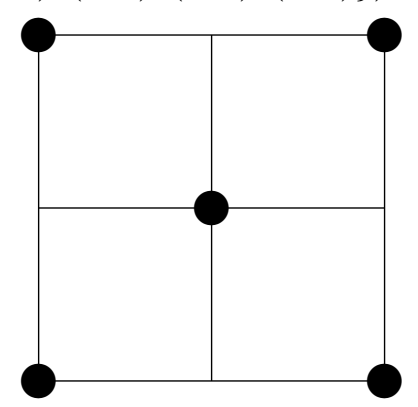

Let parametric support $S=\{(0,0),(2,0),(1,1),(0,2),(2,2)\})$. Again by the technique of random coefficients or otherwise, we find $A D(S)=4$. Thus

$$
I X(\{(0,0),(2,0),(1,1),(0,2),(2,2)\})=\frac{2 \times 2 \times 2}{4}=2 .
$$

Indeed, there are two parameter points $(\sqrt{s / t}, \sqrt{s t}),(-\sqrt{s / t},-\sqrt{s t})$ corresponding to a general surface point $(X, Y, Z)$.

\section{3..3. Example: Triangular supports $|S|=3$}

Let parametric support $S=\{(i, j),(k, l),(p, q)\}$ consist of three non-collinear lattice points. It can be easily verified that a rational parametrization on $S$ represents a plane, thus $A D(S)=1$ and

$$
I X(\{(i, j),(k, l),(p, q)\})=N A(N P(S))=a b s\left|\begin{array}{ccc}
i & j & 1 \\
k & l & 1 \\
p & q & 1
\end{array}\right| .
$$

This means the improper index of a triangular parametric support is simply its normalized area. Consequently, a triangular parametric support is improper if and only if its normalized area is greater than 1 . 


\subsection{Support Transformations are Reparametrizations}

Proposition 1 A support transformation is a reparametrization.

Proof

Let $T(i, j)=(a i+b j+c, d i+e j+f)=\left(i^{\prime}, j^{\prime}\right)$ be a support transformation for the parametric support $S$. To see the reparametrization introduced by $T$, let

$$
s=u^{a} v^{d}, \quad t=u^{b} v^{e} .
$$

We have

$$
\begin{aligned}
\frac{\sum_{(i, j) \in S}\left(x_{i, j}, y_{i, j}, z_{i, j}\right) s^{i} t^{j}}{\sum_{(i, j) \in S} w_{i, j} s^{i} t^{j}} & =\frac{\sum_{(i, j) \in S}\left(x_{i, j}, y_{i, j}, z_{i, j}\right) u^{a i+b j} v^{d i+e j}}{\sum_{(i, j) \in S} w_{i, j} u^{a i+b j} v^{d i+e j}} \\
& =\frac{\sum_{\left(i^{\prime}, j^{\prime}\right) \in T(S)}\left(x_{i, j}, y_{i, j}, z_{i, j}\right) u^{i^{\prime}} v^{j^{\prime}}}{\sum_{\left(i^{\prime}, j^{\prime}\right) \in T(S)} w_{i, j} u^{i^{\prime}} v^{j^{\prime}}}
\end{aligned}
$$

\subsection{A Shrinkable Support is Improper}

Proposition 2 If there is a support transformation $T$ for a surface parametric support $S$ with $J(T)<1$, then $S$ is an improper parametric support and

$$
\frac{I X(S)}{I X(T(S))}=\frac{1}{J(T)}
$$

Proof

By the previous proposition, a parametrization on the support $T(S)$ is a reparametrization of a parametrization on the support $S$. Thus $A D(T(S))=A D(S)$ and

$$
\frac{I X(S)}{I X(T(S))}=\frac{N A(N P(S)) A D(T(S))}{N A(N P(T(S))) A D(S)}=\frac{N A(N P(S))}{N A(N P(T(S)))}=\frac{1}{J(T)} .
$$

Since $I X(T(S)) \geq 1$ so $I X(S)>1$ and $S$ is improper.

\section{I}

2.5..1. Example: $S=\{(0,0),(2,0),(1,1),(0,2),(2,2)\}$

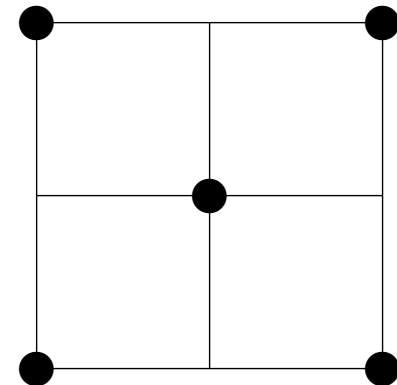

Instead of computing $A D(S)$ to find $I X(S)$ (see Example 2.3..2.), we can check that the support is improper because there is a shrinking support transformation

$$
T(x, y)=\left(\frac{x+y-2}{2}, \frac{y-x}{2}\right), \quad J(T)=1 / 2 .
$$




\section{5..2. Example: Triangular supports $|S|=3$}

In addition to the method of Example 2.3..3., we can examine if a triangular support $S=\{(i, j),(k, l),(p, q)\}$ is improper by considering the transformation

$$
T(x, y)=\left(\frac{\left|\begin{array}{ccc}
i & j & 1 \\
x & y & 1 \\
p & q & 1
\end{array}\right|}{\left|\begin{array}{ccc}
i & j & 1 \\
k & l & 1 \\
p & q & 1
\end{array}\right|}, \frac{\left|\begin{array}{ccc}
i & j & 1 \\
k & l & 1 \\
x & y & 1
\end{array}\right|}{\left|\begin{array}{ccc}
i & j & 1 \\
k & l & 1 \\
p & q & 1
\end{array}\right|}\right)
$$

Since $T(S)=\{(0,0),(1,0),(0,1)\}, T$ is a support transformation for $S$ with

$$
J(T)=a b s\left|\begin{array}{ccc}
i & j & 1 \\
k & l & 1 \\
p & q & 1
\end{array}\right|^{-1}=N A(N P(S))^{-1}=I X(S)^{-1} .
$$

The equality

$$
I X(S) J(T)=1
$$

leads to a statement stronger than Proposition 2: a triangular support is improper if and only if it is shrinkable.

\section{The GCD of Normalized Areas of Triangular Sub-Supports}

The main result of this section is

$$
I X(S)=\operatorname{gcd}\left\{N A\left(N P\left(S^{\prime}\right)\right): S^{\prime} \subseteq S,\left|S^{\prime}\right|=3\right\} .
$$

The result is significant because it computes the non-trivial improper index of a support with elementary means.

\subsection{Improper Index of a Support Divides Improper Indices of Sub-Supports}

First we show that if $S^{\prime} \subseteq S$ then $I X(S) \mid I X\left(S^{\prime}\right)$.

Proposition 3 Let $S$ be a parametric support. If $S^{\prime} \subseteq S$ then $I X(S) \mid I X\left(S^{\prime}\right)$.

Proof

The proof is based on the Factors of Zero Theorem [1]. The implicit equation of the generic surface with support $S$ has the form $f^{n}$ where $n=I X(S)$ and $f$ is a polynomial equations in $X, Y, Z$ and the generic coefficients $w_{i, j}, x_{i, j}, y_{i, j}, z_{i, j},(i, j) \in S$. To obtain $S^{\prime}$ from $S$, we set $w_{i, j}, x_{i, j}, y_{i, j}, z_{i, j}$ to zero successively for each $(i, j) \in S \backslash S^{\prime}$. At each step by factorization if necessary we extract a "fragment" of $f$ which is the implicit equation of the generic surface with support $S \backslash\{(i, j)\}$. This implicit equation thus has the form $f_{1}^{n m_{1}}$ for some integer $m_{1} \geq 1$ depending on $(i, j)$. If this is done for each $(i, j) \in S \backslash S^{\prime}$, we obtain the implicit equation for $S^{\prime}$ which has the form $f_{p}^{n m_{p}}$ for some integer $m_{p} \geq 1$. This means $I X\left(S^{\prime}\right)=n m_{p}$ and thus $I X(S) \mid I X\left(S^{\prime}\right)$.

I

The following corollaries are direct consequences of the above theorem. 
Corollary 1 Every sub-support of an improper surface parametric support is improper.

Corollary 2 If a sub-support of a surface parametric support is proper, then the support is also proper.

Corollary 3 Let $S_{1}, \ldots, S_{n}$ be sub-supports of a surface parametric support $S$. If $\operatorname{gcd}\left(I X\left(S_{1}\right), \ldots, I X\left(S_{n}\right)\right)=1$, then $S$ is a proper parametric support.

Corollary 4 Let $S_{1}, \ldots, S_{n}$ be sub-supports of a surface parametric support $S$. If $S$ is improper then $\operatorname{gcd}\left(I X\left(S_{1}\right), \ldots, I X\left(S_{n}\right)\right)>1$.

3.1..1. Example: Support $\{(1,0),(1,1),(0,1),(2,1),(1,2)\}$ is proper

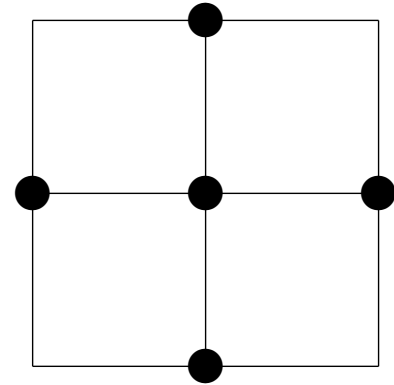

The support is proper because any three adjacent points form a proper parametric support.

3.1..2. Example: Support $\{(1,0),(2,1),(1,2),(0,1)$,$\} is proper$

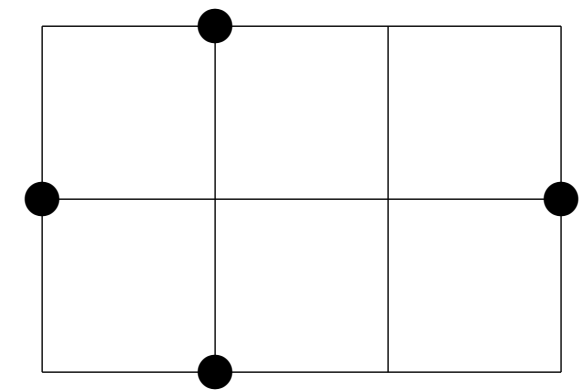

The improper indices of the bottom, right, top, left triangular sub-supports are $3,4,3$, 2 respectively. Since $\operatorname{gcd}(3,4,3,2)=1$, the given support is proper.

\subsection{The Main Result}

Now we are ready to prove the main result.

Theorem 1 Let $S$ be a surface parametric support. We have

$$
I X(S)=\operatorname{gcd}\left\{N A\left(N P\left(S^{\prime}\right)\right): S^{\prime} \subseteq S,\left|S^{\prime}\right|=3\right\} .
$$

Proof 
Without loss of generality we may assume $(0,0) \in S$. For any $(x, y),\left(x_{i}, y_{i}\right) \in S$, we have

$$
N A\left(N P\left(\left\{(0,0),(x, y),\left(x_{i}, y_{i}\right)\right\}\right)\right)=a b s\left|\begin{array}{ccc}
0 & 0 & 1 \\
x & y & 1 \\
x_{i} & y_{i} & 1
\end{array}\right|=a b s\left(y_{i} x-x_{i} y\right)
$$

Let $\operatorname{gcd}\left\{N A\left(N P\left(S^{\prime}\right)\right): S^{\prime} \subseteq S,\left|S^{\prime}\right|=3\right\}=g$. For any $(x, y),\left(x_{i}, y_{i}\right) \in S$, we can write

$$
y_{i} x-x_{i} y=g f_{i} .
$$

Let

$$
\operatorname{gcd}\left\{y_{i}:\left(x_{i}, y_{i}\right) \in S\right\}=g_{y} .
$$

There exist integers $b_{i}$ such that $\sum_{i} b_{i} y_{i}=g_{y}$. Thus

$$
\frac{g_{y} x-\left(\sum_{i} b_{i} x_{i}\right) y}{g}=\sum b_{i} f_{i}
$$

is an integer and the transformation

$$
T(x, y)=\left(\frac{g_{y} x-\left(\sum_{i} b_{i} x_{i}\right) y}{g}, \frac{y}{g_{y}}\right)
$$

is a support transformation with Jacobian $J(T)=1 / g<1$.

Since $T$ is invertible and $I X\left(S^{\prime}\right)=N A\left(N P\left(S^{\prime}\right)\right)$ when $\left|S^{\prime}\right|=3$, thus we have

$$
\begin{aligned}
\operatorname{gcd}\left\{I X\left(S^{\prime \prime}\right): S^{\prime \prime} \subseteq T(S),\left|S^{\prime \prime}\right|=3\right\} & =\operatorname{gcd}\left\{I X\left(T\left(S^{\prime}\right)\right): S^{\prime} \subseteq S,\left|S^{\prime}\right|=3\right\} \\
& =J(T) \operatorname{gcd}\left\{I X\left(S^{\prime}\right): S^{\prime} \subseteq S,\left|S^{\prime}\right|=3\right\} \\
& =\frac{\operatorname{gcd}\left\{N A\left(N P\left(S^{\prime}\right)\right): S^{\prime} \subseteq S,\left|S^{\prime}\right|=3\right\}}{g} \\
& =1
\end{aligned}
$$

By Proposition 3 we conclude $I X(T(S))=1$. But $I X(T(S))=I X(S) J(T)$ by Proposition 2, so we have $I X(S)=g$.

3.2..1. Example: $\{(0,0),(2,0),(3,3),(0,2)\}$ is improper

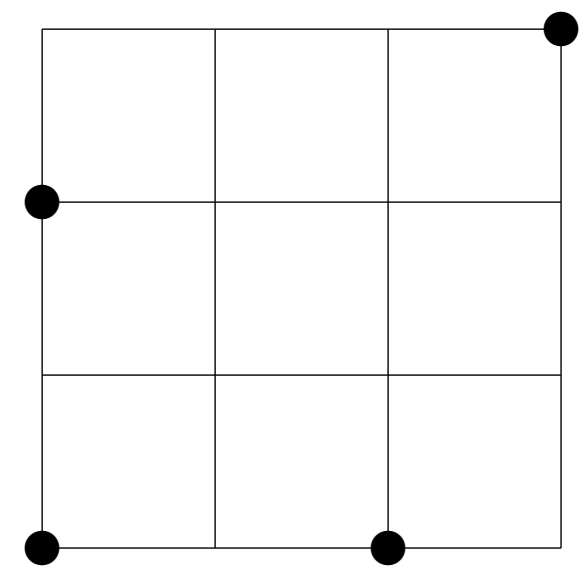


The improper indices of the four triangular sub-supports are 4, 6, 6, 8. Their gcd is 2 and thus the given support is improper.

The theorem may also be phrased in the following form:

Corollary 5 For a surface parametric support $S$, there exists a support transformation $T$ such that $I X(S) J(T)=1$ and $T(S)$ is proper.

For any four points $O, A, B, C$, the normalized area $N A(A B C)$ is a sum or difference among the values $N A(O A B), N A(O B C), N A(O A C)$, thus instead of computing the gcd of all triangular subsupports we need only compute the gcd of triangular subsupports anchored at some chosen point.

Corollary 6 Let $S$ be a surface parametric support with improper index $I X(S)$ and $(a, b) \in$ S. We have

$$
I X(S)=\operatorname{gcd}\left\{N A\left(N P\left(S^{\prime}\right)\right): S^{\prime} \subseteq S,\left|S^{\prime}\right|=3,(a, b) \in S^{\prime}\right\}
$$

Note that Corollary 6 provides an algorithm to compute $I X(S)$ with $O\left(|S|^{2}\right)$ integer gcd computations.

\subsection{Constructing an $n$-Dimensional $T$}

Perhaps the least trivial part of generalizing the results to rational parametrizations from $k^{n}$ to $k^{n+1}$ is the construction of a support transformation $T$ such that $I X(S) J(T)=1$. To generalize $T$ in Theorem 1 to $n$-dimensions, we adopt the following notations and conventions:

- $S \subseteq Z^{n}$ is a surface parametric support.

- Lattice point $P^{i}=\left(x_{1}^{i}, \cdots, x_{n}^{i}\right) \in S$ and its last $j$ coordinates projection is $P_{j}^{i}=$ $\left(x_{n+1-j}^{i}, \cdots, x_{n}^{i}\right)$.

- The normalized area of projections of lattice points of $S$ is a $(j+1) \times(j+1)$ determinant

$$
A_{j}^{\sigma}=a b s\left|\begin{array}{rr}
P_{j}^{\sigma(1)} & 1 \\
\vdots & \vdots \\
P_{j}^{\sigma(j+1)} & 1
\end{array}\right|
$$

where $\sigma$ chooses $j+1$ points from $S$.

$$
g_{j}=\underset{\sigma}{\operatorname{gcd}}\left\{A_{j}^{\sigma}\right\}, j=n, \cdots, 1 .
$$

- The question mark notation (?) in the following discussion denotes an integral quantity whose actual value is not important in the derivation.

By assuming $(0, \cdots, 0) \in S$ and mimicking the proof of Theorem 1, a support transformation $T$ with $J(T)=g_{n}$ is $T\left(x_{1}, \cdots, x_{n}\right)=$

$$
\left(\frac{x_{1} g_{n-1}+x_{2}(?)+\cdots+x_{n}(?)}{g_{n}}, \frac{x_{2} g_{n-2}+x_{3}(?)+\cdots+x_{n}(?)}{g_{n-1}}, \cdots, \frac{x_{n}}{g_{1}}\right)
$$

We state the above discussion as a theorem. 
Theorem 2 Let $g_{n}$ and $T$ be given by (29) and (30) respectively. For a parametric support $S \subseteq \mathbf{Z}^{n}, I X(S)=g_{n}$ and $T(S)$ is a proper support.

\section{Likelihood of a Surface Parametric Support to be Improper}

It is beyond the scope of the paper to describe the structure of the lattice space from which improper supports of a given improper index is a subset. Instead we examine supports, degenerate or otherwise, of popular total degree and bidegree configurations to find out how many of them are improper.

\subsection{Total Degree Supports}

A support $S \subseteq \mathbf{Z} \times \mathbf{Z}$ is considered to be total degree $n$ if

$$
\min \left\{x_{i}:\left(x_{i}, y_{i}\right) \in S\right\}=\min \left\{y_{i}:\left(x_{i}, y_{i}\right) \in S\right\}=0,
$$

and

$$
\max \left\{x_{i}+y_{i}:\left(x_{i}, y_{i}\right) \in S\right\}=n .
$$

We have the following numbers

\begin{tabular}{|c|c|c|c|}
\hline$n$ & \# of Supports & \# of Improper Supports & $\%$ \\
\hline 2 & 39 & 10 & $26 \%$ \\
3 & 845 & 118 & $14 \%$ \\
\hline
\end{tabular}

\subsection{Bidegree Supports}

A support $S \subseteq \mathbf{Z} \times \mathbf{Z}$ is considered to be bidegree degree $m \times n$ if

$$
\min \left\{x_{i}:\left(x_{i}, y_{i}\right) \in S\right\}=\min \left\{y_{i}:\left(x_{i}, y_{i}\right) \in S\right\}=0,
$$

and

$$
\max \left\{x_{i}:\left(x_{i}, y_{i}\right) \in S\right\}=m, \max \left\{y_{i}:\left(x_{i}, y_{i}\right) \in S\right\}=n .
$$

We have the following numbers

\begin{tabular}{|c|c|c|c|}
\hline$m \times n$ & \# of Supports & \# of Improper Supports & $\%$ \\
\hline $2 \times 2$ & 320 & 65 & $20 \%$ \\
$3 \times 3$ & 51470 & 501 & $1 \%$ \\
\hline
\end{tabular}

\section{Conclusion}

Instead of looking at surface rational parametrizations that are improper, we examine monomial supports from which any rational parametrization is improper. We call such an inherently improper surface parametric support an improper support. The main result of the paper is Equation (20) which we repeat below:

$$
I X(S)=\operatorname{gcd}\left\{N A\left(N P\left(S^{\prime}\right)\right): S^{\prime} \subseteq S,\left|S^{\prime}\right|=3\right\} .
$$

The significance of this formula is that it calculates the improper index of a support $S$ with elementary means and avoids the difficulty of finding the degree of the generic surface defined 
on the support. The proof of this equality can be used to detect and correct an improper parametrization due to an improper support with $O\left(|S|^{2}\right)$ integer gcd computations. Furthermore, these results can be generalized to rational parametrizations from $k^{n}$ to $k^{n+1}$ for $n>2$.

We have assessed the likelihood of encountering an improper support of some popular degree configurations and found the presence of improper supports to be quite significant. We leave the complete description of lattice spaces in which improper supports reside for future research.

Just like monomial ideals are a special but significant special case of general ideals, we hope but do not know now if the results on the special case of improper supports will be useful in the investigation of the general problem of improper parametrizations.

\section{Acknowledgements}

The first author is very grateful to the Key Laboratory of Mathematics Mechanization (KLMM), Chinese Academy of Sciences, for the supports, facilities, and hospitality during his sabbatical visit.

\section{References}

[1] Chionh E.W., Goldman R.N. Implicitizing rational surfaces with base points by applying perturbations and the factors of zero theorem. Conference on Curves, Surfaces, CAGD, and Image Processing, Biri, Norway, June 1991.

[2] Chionh E.W., Goldman R.N. Degree, multiplicity, and inversion formulas for rational surfaces using u-resultants. Computer Aided Geometric Design, 9:93-108, 1992.

[3] Cox D., Little J., O'Shea D. Using Algebraic Geometry. Springer-Verlag, New York, 1998.

[4] Gao X.S., Chou S.C. Implicitization of rational parametric equations. Journal of Symbolic Computation, 14, 459-470, 1992.

[5] Pérez-Díaz S., Schicho J., Sendra J.R. Properness and inversion of rational parametrizations of surfaces. Applicable Algebra in Engineering Communication and Computing, 13, 29-51, 2002.

[6] Schinzel A. Polynomials with Special Regard to Reducibility. Cambridge University Press, 2000.

[7] Sederberg T.W. Improperly parametrized rational curves. Computer Aided Geometric Design, 3, 67-75, 1986.

[8] Sommerville D.M.Y. Analytical Geometry of Three Dimensions. Cambridge University Press, 1959. 